\title{
The EURITRACK project: development of a tagged neutron inspection system for cargo containers.
}

\author{
Bertrand Perot $^{*}$, , Gregory Perret ${ }^{a}$, Alain Mariani ${ }^{a}$, Jean-Luc Ma ${ }^{a}$, Jean-Louis Szabo $^{b}$, \\ Emmanuel Mercier ${ }^{b}$, Guillaume Sannie $^{b}$, Giuseppe Viesti ${ }^{c}$, Giancarlo Nebbia ${ }^{c}$, Silvia Pesente ${ }^{c}$, \\ Marcello Lunardon ${ }^{c}$, Paola Formisano ${ }^{c}$, Sandra Moretto ${ }^{c}$, Daniela Fabris ${ }^{c}$, Aldo Zenoni ${ }^{d}$, \\ Germano Bonomi $^{d}$, Antonietta Donzella ${ }^{d}$, Andrea Fontana ${ }^{e}$, Gaia Boghen $^{e}$, Vladivoj Valkovic ${ }^{f}$, \\ Davorin Sudac ${ }^{\prime}$, Marek Moszynski ${ }^{g}$, Tadeusz Batsch ${ }^{g}$, Michal Gierlik $^{g}$, Dariusz Wolski ${ }^{g}$, \\ Wlodzimierz Klamra $^{h}$, Patrick Isaksson ${ }^{h}$, Philippe Le Tourneur ${ }^{i}$, Miguel Lhuissier ${ }^{i}$, \\ Annamaria Colonna ${ }^{j}$, Carlo Tintori ${ }^{j}$, Paolo Peerani ${ }^{k}$, Vitor Sequeira $^{k}$, Martino Salvato $^{k}$ \\ ${ }^{a}$ Commissariat à l'Energie Atomique, 13109 St Paul-lez-Durance Cedex, France; \\ ${ }^{b}$ Commissariat à 1'Energie Atomique, 91191 Gif-Sur-Yvette Cedex, France; \\ ${ }^{c}$ INFN and Università di Padova, Via Marzolo 8, I-35131 Padova, Italy; \\ ${ }^{d}$ INFN and Università di Brescia, Via Branze 38, 25123 Brescia, Italy; \\ ${ }^{e}$ INFN and Università di Pavia, Via Bassi 6, 27100 Pavia, Italy; \\ ${ }^{f}$ Institute Ruder Boskovic, 54 Bijenicka c. 10000 Zagreb, Croatia \\ ${ }^{g}$ Soltan Institute for Nuclear Studies, PL 05-400 Otwock-Swierk, Poland \\ ${ }^{h}$ Department of Physics Alba Nova of Royal Institute of Technology,10691 Stockholm, Sweden \\ ${ }^{i}$ EADS-SODERN, 20 Av. Descartes 94451 Limeil-Brévannes Cedex, France \\ ${ }^{j}$ CAEN S.p.A., Via Vetraia 11, 55049 Viareggio (LU), Italy \\ ${ }^{k}$ European Commission - Joint Research Centre IPSC - CCR, I-21020 Ispra (VA), Italy
}

\begin{abstract}
The EURopean Illicit TRAfficing Countermeasures Kit project is part of the 6th European Union Framework Program, and aims at developing a neutron inspection system for detecting threat materials (explosives, drugs, etc.) in cargo containers. Neutron interaction in the container produces specific gamma-rays used to determine the chemical composition of the inspected material. An associated particle sealed tube neutron generator is developed to allow precise location of the interaction point by direction and time-of-flight measurements of the neutrons tagged by alpha-particles.

The EURITRACK project consists in developing: a transportable deuterium-tritium neutron generator including a position sensitive alpha detector ( $8 \times 8$ matrix of YAP:Ce crystals coupled to a multi-anode photomultiplier), fast neutron and gamma-ray detectors, front-end electronics to perform coincidence and spectroscopic measurements, and an integrated software which manages neutron generator and detectors positioning, data acquisition and analysis. Hardware components have been developed and tested by the consortium partners. Current status of this work and provisional performances of the system assessed by Monte Carlo calculations are presented.
\end{abstract}

Keywords: EURITRACK, Civil Security, Neutron Inspection, Associated Particle Technique, Cargo Container

\section{INTRODUCTION}

Fast, non-intrusive inspection of cargo containers has become a key issue in recent years to parry terrorist activities. Controls are mainly based on X- or gamma-ray radiography which allows determining the shape and density of goods inside containers but not their elemental composition. Fast Neutron Analysis (FNA) technique can be additionally used to deduce the elemental ratios of carbon, oxygen and nitrogen from the relative intensity of their gamma-ray lines ${ }^{1,2,3}$.

\footnotetext{
* Further author information: (Send correspondence to Bertrand Perot.)

Bertrand Perot.: E-mail: bertrand.perot@cea.fr, Telephone: +33 442254048
}

Non-Intrusive Inspection Technologies, edited by George Vourvopoulos, F. Patrick Doty, Proc. of SPIE Vol. 6213, 621305, (2006) - 0277-786X/06/\$15 · doi: 10.1117/12.665380 
The EURITRACK project aims at developing a non-intrusive Tagged Neutron Inspection System (TNIS) based on the Associated Particle Technique (APT) ${ }^{4}$. The neutron generator produces $14-\mathrm{MeV}$ neutrons and alpha particles from deuterium-tritium interactions. Alpha particles and neutrons are emitted back-to-back, simultaneously. A portion of the alpha particles are detected by a $8 \times 8$ matrix of YAP:Ce crystals enabling to tag a portion of the neutrons in terms of direction and time. In practice, a sealed tube neutron generator with a built-in segmented alpha particle detector is used to interrogate a delimited volume of the container. Appropriate detectors and electronics allow measuring coincidence between alpha particles and gamma-rays with nanosecond resolution. The speed of $14-\mathrm{MeV}$ neutrons is approximately $5 \mathrm{~cm} / \mathrm{ns}$, while the spatial resolution of the TNIS is a few dozens centimeters.

The gamma-ray spectrum induced by fast neutron interactions on materials inside the suspect area characterizes their relative amount of carbon, oxygen and nitrogen. The 4439-, 6130-, and 5106- \& 2313-keV gamma-ray lines are the most useful for carbon, oxygen and nitrogen determination, respectively.

\section{TNIS GENERAL DESIGN AND CALCULATED PERFORMANCES}

Monte Carlo calculations were performed using $\mathrm{MCNP}^{5}$ to optimize the general design of the TNIS: neutron generator and detectors positions, shields and collimators. Neutron and photon fluxes at the entrance of the detectors, the associated useful signal and the signal-to-noise ratio were determined ${ }^{6}$.

The general layout of the TNIS is shown in Fig. 1. The deuterium-tritium neutron tube emits $10^{8} \mathrm{n} / \mathrm{s}$ isotropically. Approximately $1 \%$ of the neutrons are tagged by detecting the alpha particles with an $8 \times 8$ matrix of YAP:Ce crystals coupled to a multi-anode photomultiplier (APT). Neutron transmission measurement is performed using a BC501A liquid scintillator in "transmission" position. Gamma-rays are detected using the two "reflection" 5 " $\times 5$ " $\mathrm{NaI}(\mathrm{Tl})$, four "transmission" $5 " \times 5 " \times 10$ " $\mathrm{NaI}(\mathrm{Tl})$ and sixteen "top" $5 " \times 5 " \times 10$ " $\mathrm{NaI}(\mathrm{Tl})$ detectors. Reflection detectors are protected from direct neutron irradiation by iron and lead shield plates. Transmission and top detectors can be positioned at different depths in lead collimators to improve signal-to-noise ratio.

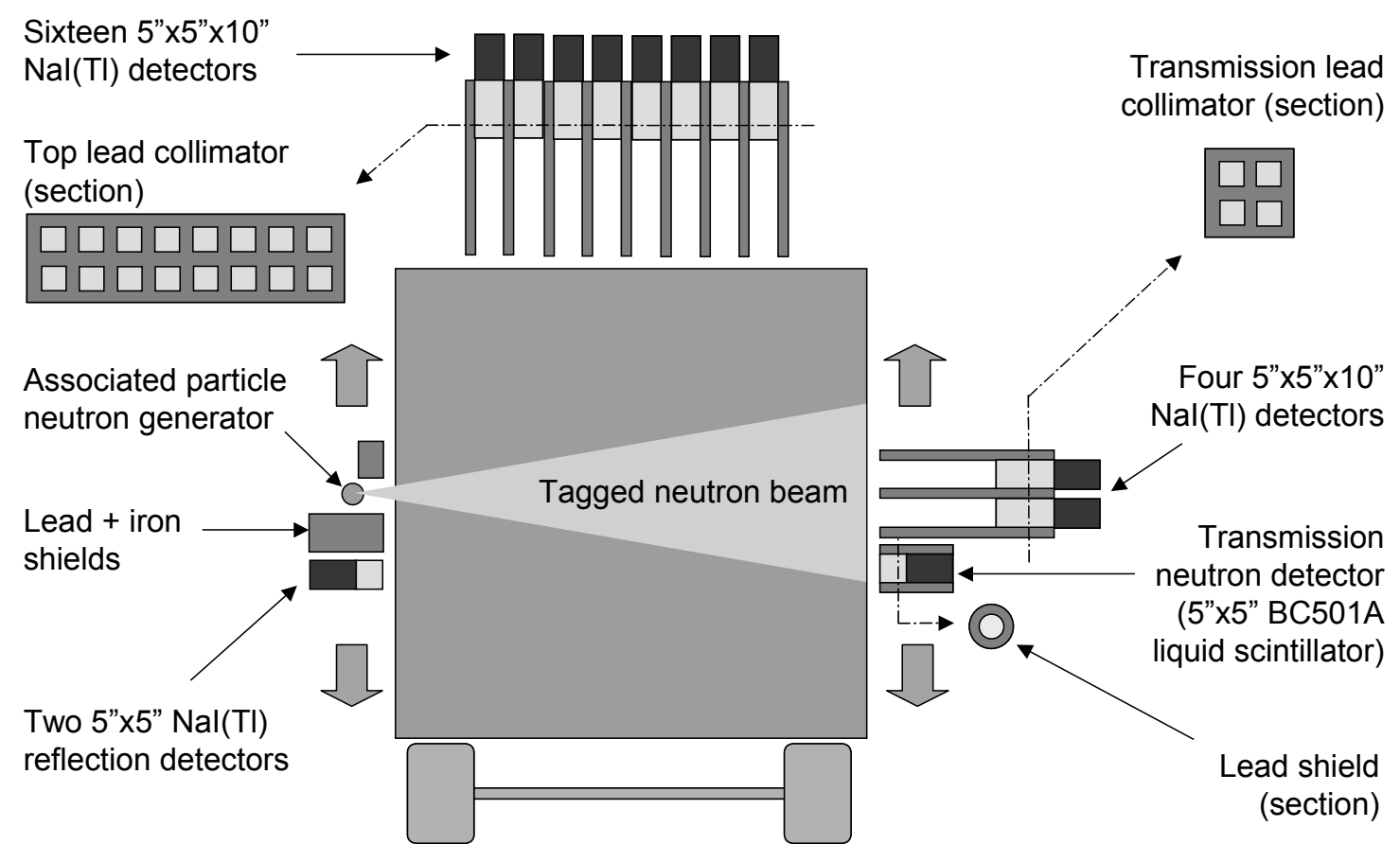

Figure 1. Tagged Neutron Inspection System set-up. Gamma-ray NaI(Tl) detectors are in top, transmission and reflection position. A BC01A neutron detector is located in a transmission position. 
Performances of the TNIS are calculated for the reference case of the EURITRACK project, which is a 100-kg block of TNT explosive located in the centre of a homogeneous metallic matrix of density $0.2 \mathrm{~g} / \mathrm{cm}^{3}$. The TNT is localized in depth using the alpha-gamma coincidence time profiles (see Fig. 2). A lower energy threshold of $1.5 \mathrm{MeV}$ is used and a TNT time window $\Delta \mathrm{t}$ is defined. The count rates within $\Delta \mathrm{t}$ are estimated using the detector entrance surface and a minimum intrinsic efficiency of 0.3 . For example, the count rates in the sixteen top detectors are approximately $80 \mathrm{cps}$ and $50 \mathrm{cps}$ with and without TNT, respectively. TNT is clearly localized in a 10-minute long irradiation.

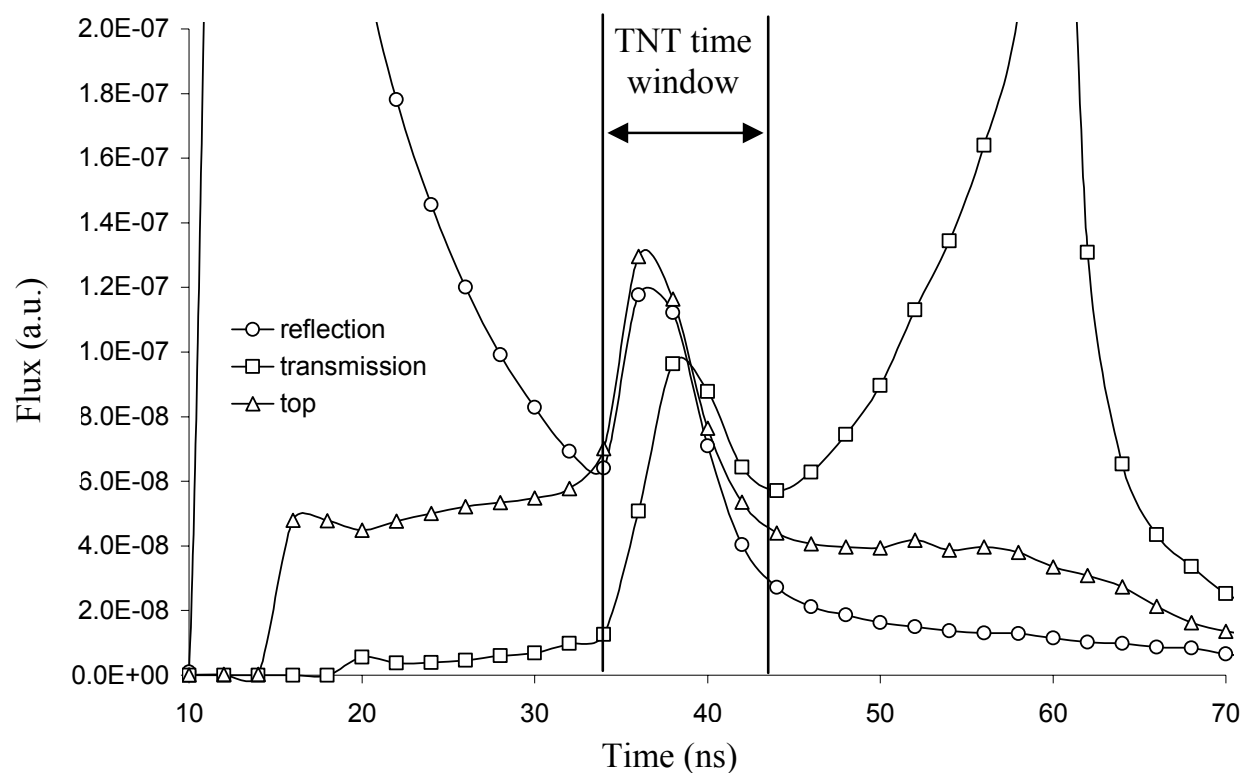

Figure 2. Time profile of the photon flux above $1.5 \mathrm{MeV}$ for the $100-\mathrm{kg}$ TNT sphere in the $0.2 \mathrm{~g} / \mathrm{cm}^{3}$ density metallic matrix. Results averaged on the top, transmission and reflection detectors.

The 4439-, 6130-, and 5106- \& 2313-keV gamma-ray lines count rate within $\Delta \mathrm{t}$ are further deduced and carbon-tooxygen and carbon-to-nitrogen ratios are calculated. For example, the carbon-to-oxygen ratios in the top and reflection detectors are $1.69 \pm 0.02$ and $1.73 \pm 0.08$, where statistical uncertainties are expressed at one standard deviation for a 10minute long irradiation. They agree within one standard deviation with the $1.75 \pm 0.08$ reference carbon-to-oxygen ratio calculated without iron matrix.

Explosives identification inside containers filled with organic goods (food, clothes, etc.) is more complex because of strong neutron attenuation and time correlated background in the organic matrix. To localize the explosive, we use the direction of the incident tagged neutron and the time difference between alpha and gamma-ray detections and draw a coincident gamma-ray flux map of the container. For example, a map of the total photon fluxes above $1.5 \mathrm{MeV}$ is shown in figure 3 for a $25-\mathrm{kg}$ TNT sphere located within an organic matrix of density $0.5 \mathrm{~g} / \mathrm{cm}^{3}$. For calculation purpose, the tracker has been made cylindrical. The position in the plan perpendicular to the tagged neutron beam axis is labeled by the radius to the tagged neutron beam axis. 


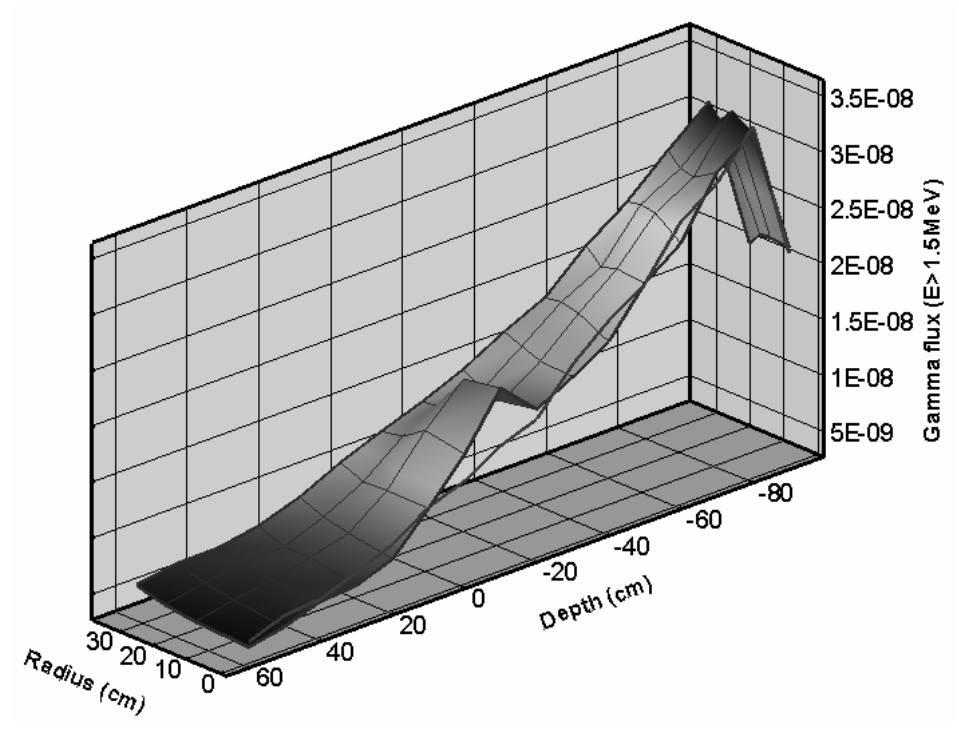

Figure 3. Coincident gamma-ray map of the container for a $25-\mathrm{kg}$ TNT sphere centered at depth $-25 \mathrm{~cm}$ in a $0.5-\mathrm{g} / \mathrm{cm}^{3}$ organic matrix (top detectors). A lower energy threshold of $1.5 \mathrm{MeV}$ is used. Radius is expressed relative to the tagged neutron beam axis and the depth origin is in the center of the container. Neutron source and $1^{\text {st }}$ container wall are at depth -175 and $-120 \mathrm{~cm}$, respectively.

\section{ASSOCIATED PARTICLE SEALED TUBE NEUTRON GENERATOR}

The SODERN associated particle sealed tube (see Fig. 4) produces $14-\mathrm{MeV}$ neutrons via the ${ }^{3} \mathrm{H}\left({ }^{2} \mathrm{H}, \mathrm{n}\right){ }^{4} \mathrm{He}$ fusion reaction. Deuterium ions are generated by a Penning ion source and accelerated before colliding with a tritiated target. The target is inclined at 45 degrees relatively to the deuteron beam to allow alpha particle detection in a matrix of YAP:Ce crystals sealed in the tube.
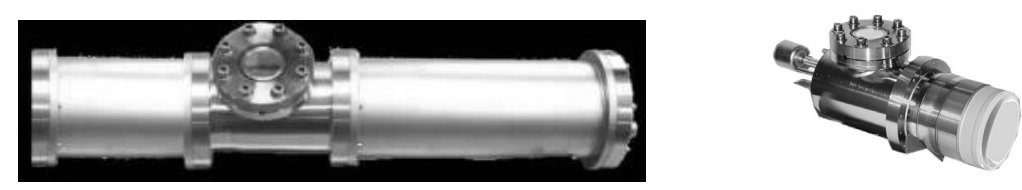

Figure 4. Housing (left) of the associated particle sealed tube neutron generator (right) manufactured by SODERN.

The alpha detector ${ }^{6}$ is composed of a stainless steel grid holding an $8 \times 8$ array (pitch of $6 \mathrm{~mm}$ ) of YAP:Ce crystals (see Fig. 5). YAP:Ce scintillators are selected for their fast response, high light output, excellent radiation hardness, and mechanical and chemical properties. The YAP:Ce crystals have a surface of $5.8 \times 5.8 \mathrm{~mm}^{2}$ and a thickness of $0.5 \mathrm{~mm}$ each. They are coupled to the flat panel Hamamatsu H8500 multi-anode photomultiplier (PM) through a 3-mm thick sapphire window mounted on an optical flange. Optical grease is not used because of the sealed neutron generator manufacturing process. The YAP:Ce crystals are coated with a $1-\mathrm{mg} / \mathrm{cm}^{2}$ silver layer to maximize light collection, stop elastically scattered deuterons and protect the crystal from UV glow inside the neutron generator.

YAP crystals are line-up with the sectors of the H8500 PM. However, because of the sapphire window, light created in crystals diffuse through the window to the adjacent sectors of the PM. To ascertain accurate positioning of the alpha particle, we used an ${ }^{241} \mathrm{Am}$ source with a mask allowing irradiation of a single YAP:Ce crystal, and studied the PM pulse-height spectra. Pixel \#36 is directly irradiated through the collimator and is encircled in the left sketch of Fig. 6. Other pixels of interest are marked. Pulse-height spectra of pixels \#36, 28, 43 and 34 are shown in the right part of Fig. 6. Energy spectra of PM \#36 and adjacent PMs do not superpose. Therefore an appropriate energy threshold permits to isolate the signal of PM \#36. "Optical cross-talk" between adjacent elements of the YAP matrix can be made negligible by energy threshold selection so that the alpha particle localization is ensured without decreasing detection efficiency. 

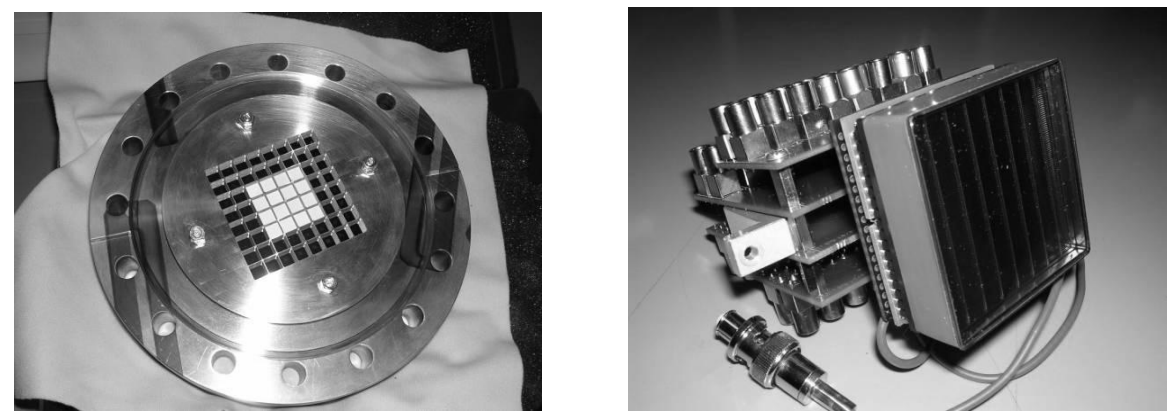

Figure 5. Alpha particle detector : YAP:Ce crystals mounted on a stainless steel grid (left) and Hamamatsu H8500 multi-anode photomultiplier with LEMO connectors mounted on a printed board (right).
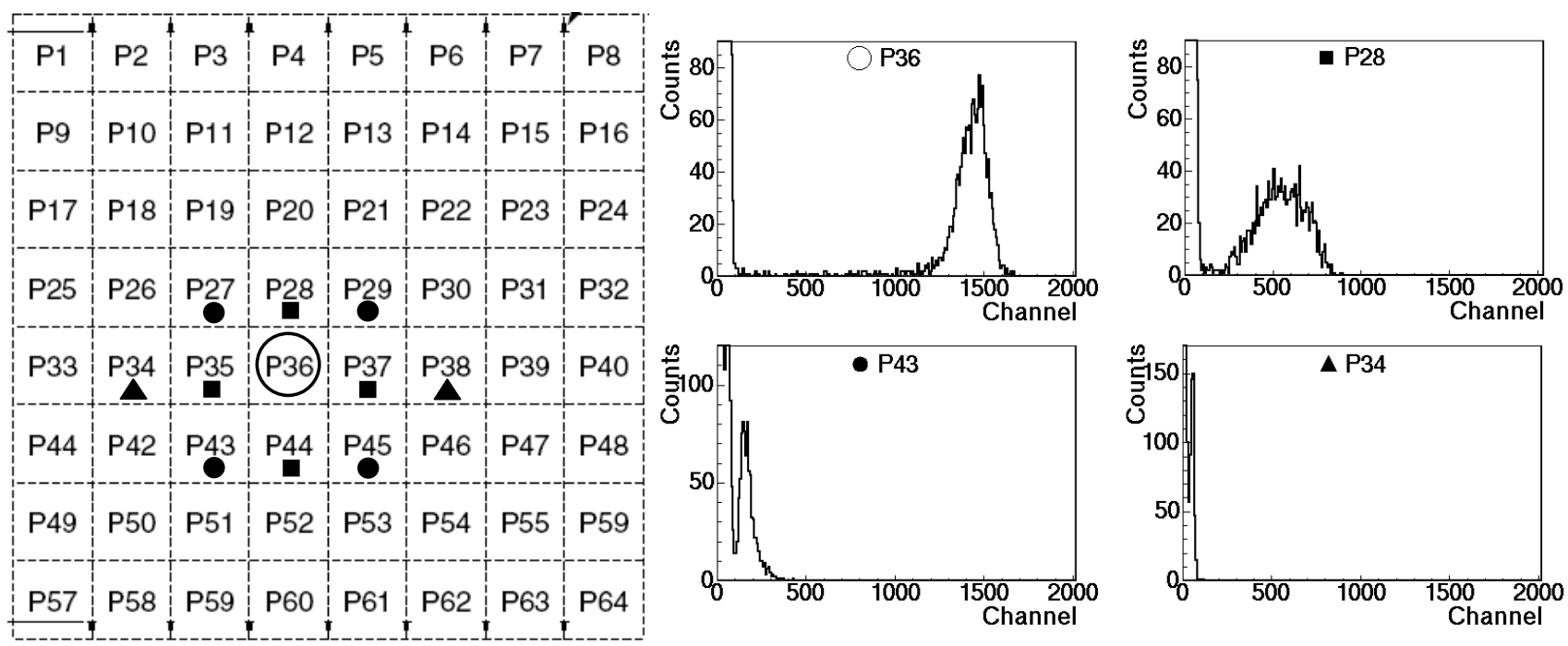

Figure 6. Alpha particle YAP:Ce crystals and energy spectra of interest.

\section{NEUTRON DETECTOR}

Neutron attenuation in the inspected container is assessed by measurement of the transmitted 14-MeV neutron flux. The neutron detector should allow $n-\gamma$ pulse shape discrimination and have a good time resolution and detection efficiency. Pulse shape discrimination is required because of the large portion of counts due to photons created in the container. Nanosecond time resolution allows reducing the coincident time window and effectively discriminate scattered neutrons from transmitted 14-MeV neutrons. Neutron efficiency of the detector is selected to allow measurements for high attenuating matrixes (e.g. organic goods) but also for empty containers, where high count rates can be detrimental to the $\mathrm{n}-\gamma$ pulse shape discrimination ${ }^{8}$.

A 5" $\times 5 "$ cylindrical BC501A liquid scintillator (see Fig. 7), manufactured by Saint-Gobain Crystals and Detectors, was selected on a basis of extensive studies carried out during the last 20 years ${ }^{9,10}$ thanks to its excellent $n-\gamma$ pulse shape capability. Its timing property is excellent because of the fast light pulse that characterizes organic scintillators. Its size provides adequate detection efficiency without deterioration of the time resolution. A fast photomultiplier tube (PMT) with low electron transit time spread and adapted geometry is essential to maintain good time resolution. The XP4512B PMT from Photonis was selected from comparative tests on 5" diameter PMTs based on its good photoelectron 
collection efficiency ${ }^{11}$. The transistorized C type voltage divider VD105K/01 from Photonis was finally chosen because of the expected high count rates in the EURITRACK inspection system.

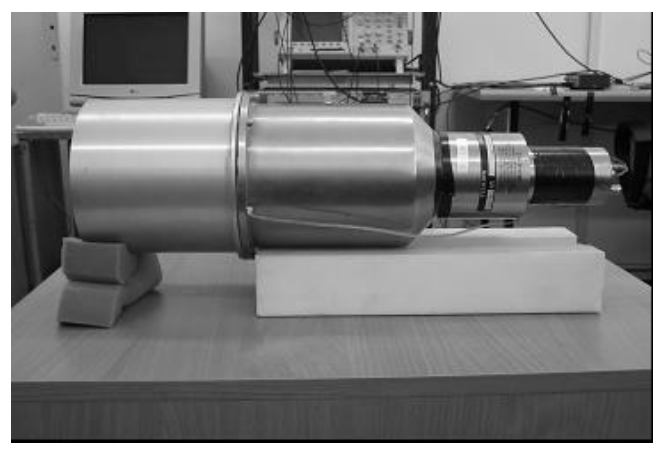

Figure 7. Neutron detector: 5" $\times 5$ " BC501A liquid scintillator coupled to a 5" diameter XP4512B PMT.

Neutron-gamma discrimination tests were performed with the selected detector and a standard Am-Be source. The reference detector for the time-of-flight measurement consisted of a $25 \times 15 \mathrm{~mm}^{2}$ barium fluoride $\left(\mathrm{BaF}_{2}\right)$ crystal coupled to a Photonis XP20Y0Q/DA fast PMT, located about $20 \mathrm{~cm}$ away from the liquid scintillator. 2D plots, using zerocrossing time of the pulse shape discrimination (labeled ZC), neutron time-of-flight (labeled TOF) and pulse height (labeled energy) are presented in Fig. 8. Excellent separation of neutron and gamma-rays is observed, particularly on the ZC time vs. TOF plot where the intense circular spot corresponds to gamma-rays. A large contribution of scattered neutrons can be noticed. For example, the TOF spectrum shows a sharp Gaussian peak corresponding to gamma-rays, and a second peak corresponding to neutrons, which tail signs the scattered neutrons (inversed time scale).
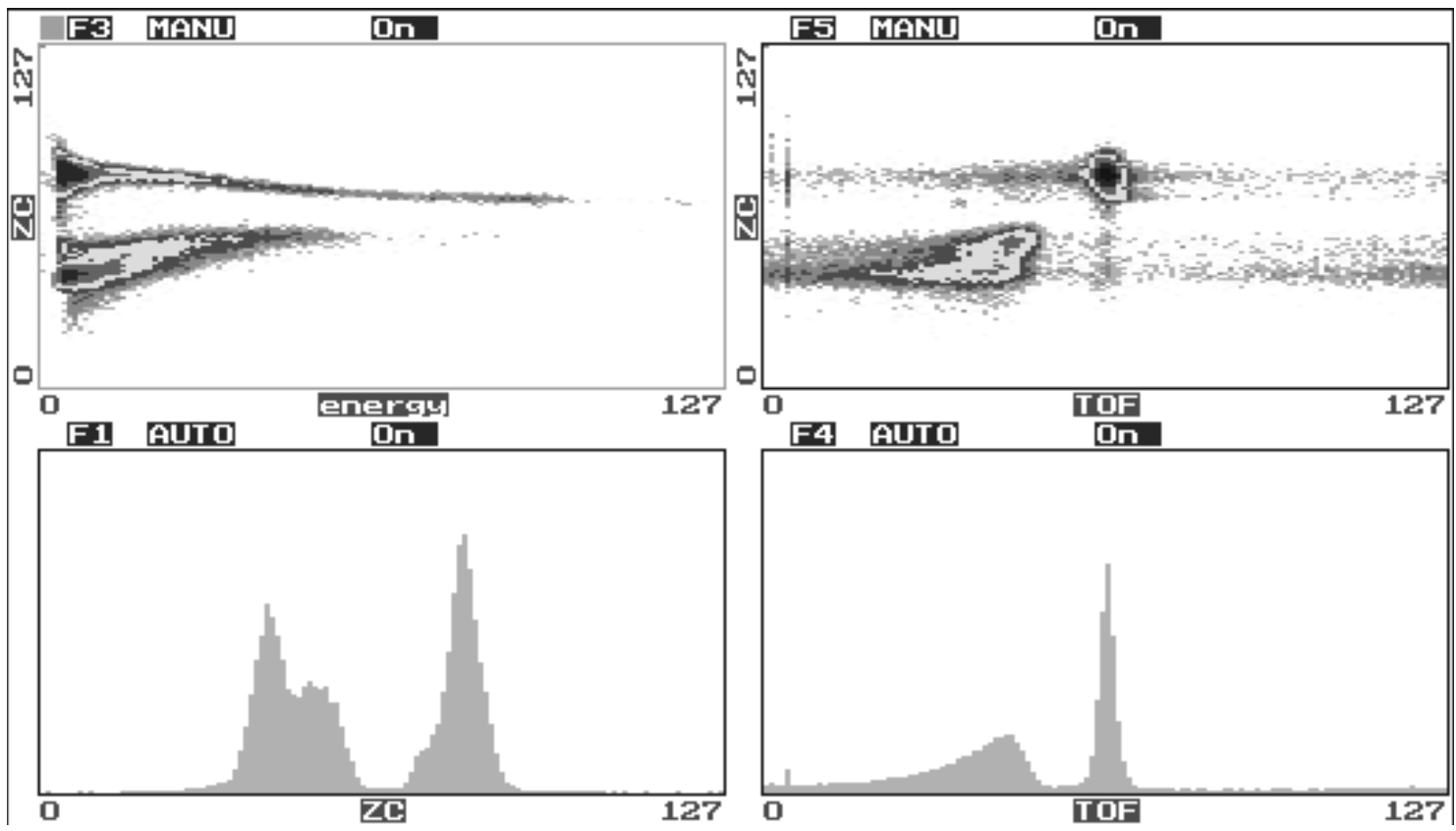

Figure 8. Neutron-gamma discrimination. 


\section{GAMMA-RAY DETECTORS}

Gamma-ray detectors are located around the container to maximize the covered solid angle (see Fig. 1 in Sec. 2). They should have large intrinsic efficiencies for high-energy gamma-rays (above $1.5 \mathrm{MeV}$ thresholds), good energy and time resolution, and a low cost. Large volume of inorganic scintillators with high density and atomic number best fulfill all these requirements.

Best candidates are barium fluoride $\left(\mathrm{BaF}_{2}\right)$, bismuth germanate $(\mathrm{BGO})$ and sodium iodide $(\mathrm{NaI}(\mathrm{Tl}))$ inorganic scintillators. Energy deposition characteristics of high-energy photons in standard and large size crystals were calculated with MCNP. The full-energy peak effective efficiency (intrinsic efficiency times detector entrance surface) is shown in Fig. 9. Full-energy peak intrinsic and effective efficiencies, and full-energy-peak-to-total ratios are listed in Tab. 1 for the 4439-keV gamma-ray line of carbon.

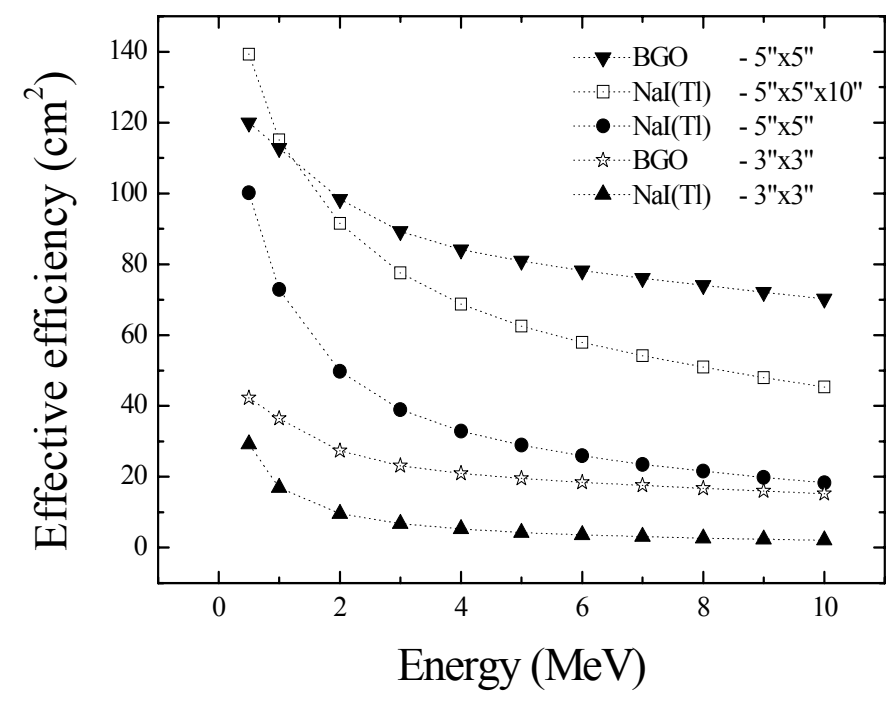

Figure 9. Full-energy peak effective efficiency for large inorganic scintillators calculated by Monte Carlo. The effective efficiency is the intrinsic efficiency times the entrance surface of the detector.

Table 1. Example of large inorganic scintillator characteristics for the 4439-keV gamma-ray line of carbon. The effective efficiency is the intrinsic efficiency times the entrance surface of the detector.

\begin{tabular}{|c|c|c|c|}
\hline Scintillator & $\begin{array}{c}\text { Intrinsic } \\
\text { full-energy peak } \\
\text { efficiency } \\
\text { (count/photon) }\end{array}$ & $\begin{array}{c}\text { Effective } \\
\text { full-energy peak } \\
\text { efficiency } \\
\left(\mathrm{cm}^{2}\right)\end{array}$ & $\begin{array}{l}\text { Peak-to-total } \\
\text { ratio }\end{array}$ \\
\hline $3^{\prime \prime} \times 3 " \mathrm{BaF}_{2}{ }^{\mathrm{a}}$ & 0.17 & 7.9 & 0.40 \\
\hline $3^{\prime \prime} \times 3^{\prime \prime} \mathrm{BGO}^{\mathrm{a}}$ & 0.44 & 20 & 1.43 \\
\hline $3^{\prime \prime} \times 3^{\prime \prime} \mathrm{NaI}(\mathrm{Tl})^{\mathrm{a}}$ & 0.10 & 4.7 & 0.25 \\
\hline $5^{\prime \prime} \times 5^{\prime \prime} \mathrm{BGO}^{\mathrm{a}}$ & 0.65 & 83 & 3.02 \\
\hline $5^{\prime \prime} \times 5^{\prime \prime} \mathrm{NaI}(\mathrm{Tl})^{\mathrm{a}}$ & 0.24 & 31 & 0.55 \\
\hline $5^{\prime \prime} \times 5^{\prime \prime} \times 10^{\prime \prime} \mathrm{NaI}(\mathrm{Tl})^{\mathrm{b}}$ & 0.39 & 66 & 0.88 \\
\hline
\end{tabular}

a) Cylindrical shape with identical diameter and length: 3 " $(7.62 \mathrm{~cm})$ or 5 " $(12.7 \mathrm{~cm})$

b) Rectangular shape: $12.7 \mathrm{~cm} \times 12.7 \mathrm{~cm} \times 25.4 \mathrm{~cm}$

BGO obviously offers the best properties for identical size, however, large $\mathrm{NaI}(\mathrm{Tl})$ crystals is a cheaper alternative solution. Within the available budget, maximum detection efficiency is to be preferred. $\mathrm{BaF}_{2}$ has lower detection efficiency than $\mathrm{BGO}$ and is as expensive. Moreover, the sub-nanosecond time resolution of $\mathrm{BaF}_{2}$ is not of any use for the 
EURITRACK project because the required spatial resolution of a dozen of centimeters is achievable with nanosecond resolution.

Time and energy resolution of available $\mathrm{NaI}(\mathrm{Tl})$ and $\mathrm{BGO}$ detectors, and additional ones purchased for the EURITRACK project, such as the large NaI(Tl) detectors (see Fig. 10), were measured ${ }^{12}$. The Photonis XP4312B and XP4512B PMTs were chosen for the 3" and 5" crystals, respectively. These linear-focused design tubes are characterized by excellent timing properties and are well suited for gamma-ray spectrometry. The Cyberstar 5 " $\times 5$ " BGO was however tested with its native R877 PMT, equipped with a venetian-blind dynode structure and a green extended photocathode. When compared to the $3 " \times 3 "$ BGO crystal coupled with Photonis PMT, we observed a considerable improvement in energy resolution at the cost of time resolution.

Measured time and energy resolutions are listed in Tab. 2 for combinations of applied PMTs and scintillators. These parameters are relatively well known for small volume detectors. Tests were therefore focused on the performances of large volume crystals. They were performed using standard ${ }^{137} \mathrm{Cs},{ }^{60} \mathrm{Co}$ and $\mathrm{Pu}$-Be sources. The reference detector used for the $\gamma-\gamma$ and $\gamma$-n coincidence measurements with the ${ }^{60} \mathrm{Co}$ and $\mathrm{Pu}-\mathrm{Be}$ sources is the same fast $\mathrm{BaF}_{2}$ detector used for the neutron detector time resolution measurement (see Sec. 4). Its time resolution of $90 \pm 4 \mathrm{ps}^{13}$, measured for the $\gamma-\gamma$ coincidences in ${ }^{60} \mathrm{Co}$, is negligible compared to the time resolution of the tested detectors.

Figure 11 illustrates the excellent time and energy resolutions of the largest $5^{\prime \prime} \times 5^{\prime \prime} \times 10^{\prime \prime} \mathrm{NaI}(\mathrm{Tl})$ detector. The time spectrum shows the $\gamma-\gamma$ and $\gamma$-n coincidence peaks obtained with the Pu-Be source. $\gamma-\gamma$ coincidences were measured between various transitions, mostly in ${ }^{12} \mathrm{C}$, and of energies roughly between $0.5 \mathrm{MeV}$ and $4.44 \mathrm{MeV}$. The capital contribution, however, to the left, narrow peak comes from $\gamma$-n coincidences, when the neutron was detected by the $\mathrm{BaF}_{2}$ reference detector, which was placed in the immediate vicinity of the Pu-Be source. The broader delayed peak is related to events in which neutrons traveled all the way to and got detected in the tested gamma detector.

Performances of the $5^{\prime \prime} \times 5^{\prime \prime}$ and $5^{\prime \prime} \times 5^{\prime \prime} \times 10^{\prime \prime} \mathrm{NaI}(\mathrm{Tl})$ detectors are very satisfactory. The energy resolution of the $5^{\prime \prime} \times 5^{\prime \prime} \times 10^{\prime \prime}$ and $3 " \times 3 " \mathrm{NaI}(\mathrm{Tl})$ detectors are comparable. The light collection from $5^{\prime \prime} \times 5^{\prime \prime} \times 10^{\prime \prime}$ crystals is thus very good and uniform even if its exit surface is bigger than the 5 " diameter PMT entrance window. Except for the 5 " $\times 5$ " BGO, all detectors demonstrate a very good time resolution of less than or slightly above $2 \mathrm{~ns}$, both for precisely selected transitions in ${ }^{60} \mathrm{Co}$ and for wider spectrum coincidences in ${ }^{12} \mathrm{C}$ with threshold set above $511 \mathrm{keV}$.

The bad time resolution of the $5 " \times 5$ " BGO is due to the coupled R877 photomultiplier. The design of venetian-blind dynodes introduces a large time spread that was also observed in the slow rise time of anode pulse of several tenths of nanoseconds. A significant improvement in the timing performance of the BGO equipped detector can be achieved by fitting it with the Photonis XP45D2B PMT with a green-extended photocathode and a screening grid mounted at the anode $^{14}$. With the actual R877 PMT, which holds a green extended photocathode, energy resolution at $4.44 \mathrm{MeV}$ is only slightly worse than that of $\mathrm{NaI}(\mathrm{Tl})$ detectors. Additional tests will be performed within the next few months with the new XP45D2B PMT to check if the 5" $\times 5 "$ BGO detector fits the EURITRACK project fast timing requirements. Eventually, the large stopping power of the BGO crystal makes it an interesting solution for the future industrial measurement system.

For now, the $5 " \times 5 " \times 10^{\prime \prime} \mathrm{NaI}(\mathrm{Tl})$ crystal equipped with XP4512B PMT is chosen as the reference detector for the TNIS demonstrator which is to be mounted during year 2006. Twenty such detectors are going to be supplied, as well as two smaller 5" $\times 5 " \mathrm{NaI}(\mathrm{Tl})$ detectors equipped with the same PMT for the use near the neutron generator. 

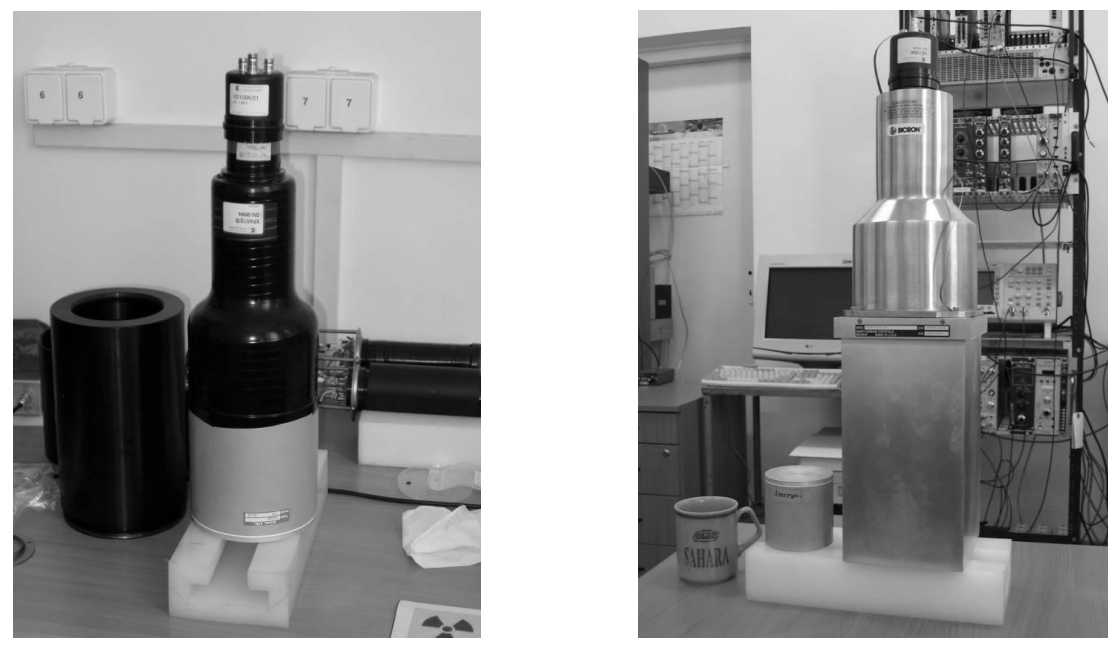

Figure 10. Large volume $\mathrm{NaI}(\mathrm{Tl})$ detectors purchased for the EURITRACK project: $5 " \times 5 "$ (left) and $5 " \times 5 " \times 10 "$ (right) crystals, both with XP4512B PMT.

Table 2. Time and energy resolutions of tested gamma-ray detectors

\begin{tabular}{|c|c|c|c|c|c|}
\hline Scintillator & PMT & $\begin{array}{l}\text { Timing } \gamma-\gamma \\
\text { in }{ }^{60} \mathrm{Co}(\mathrm{ns})\end{array}$ & $\begin{array}{l}\text { Timing } \gamma \text {-n } \\
\text { in }{ }^{12} \mathrm{C}(\mathrm{ns})\end{array}$ & $\begin{array}{c}\text { FWHM } \\
@ 662 \mathrm{keV} \\
(\%)\end{array}$ & $\begin{array}{c}\text { FWHM } \\
\text { @ } 4.44 \mathrm{MeV} \\
(\%)\end{array}$ \\
\hline Amcrys-H - NaI(Tl) 3"×3" & XP4312B & $0.89 \pm 0.08$ & $1.14 \pm 0.03$ & $7.30 \pm 0.10$ & $4.24 \pm 0.07$ \\
\hline Scionix Holland BV - BGO 3"×3" & XP4312B & $1.91 \pm 0.05$ & $1.90 \pm 0.05$ & $13.35 \pm 0.09$ & $6.20 \pm 0.06$ \\
\hline Amcrys-H - NaI(Tl) 5" $\times 5 "$ & XP4512B & $1.44 \pm 0.04$ & $1.61 \pm 0.05$ & $8.00 \pm 0.08$ & $4.87 \pm 0.03$ \\
\hline Saint-Gobain $-\mathrm{NaI}(\mathrm{Tl}) 5^{\prime \prime} \times 5^{\prime \prime}$ & XP4512B & $1.02 \pm 0.09$ & $1.80 \pm 0.04$ & $6.92 \pm 0.09$ & $3.86 \pm 0.06$ \\
\hline Cyberstar - BGO 5"×5" & R877 & $6.68 \pm 0.17$ & $5.59 \pm 0.14$ & $11.32 \pm 0.07$ & $5.14 \pm 0.08$ \\
\hline Saint-Gobain $-\mathrm{NaI}(\mathrm{Tl}) 5^{\prime \prime} \times 5^{\prime \prime} \times 10^{\prime \prime}$ & XP4512B & $1.48 \pm 0.03$ & $2.13 \pm 0.04$ & $7.39 \pm 0.06$ & $4.62 \pm 0.05$ \\
\hline
\end{tabular}
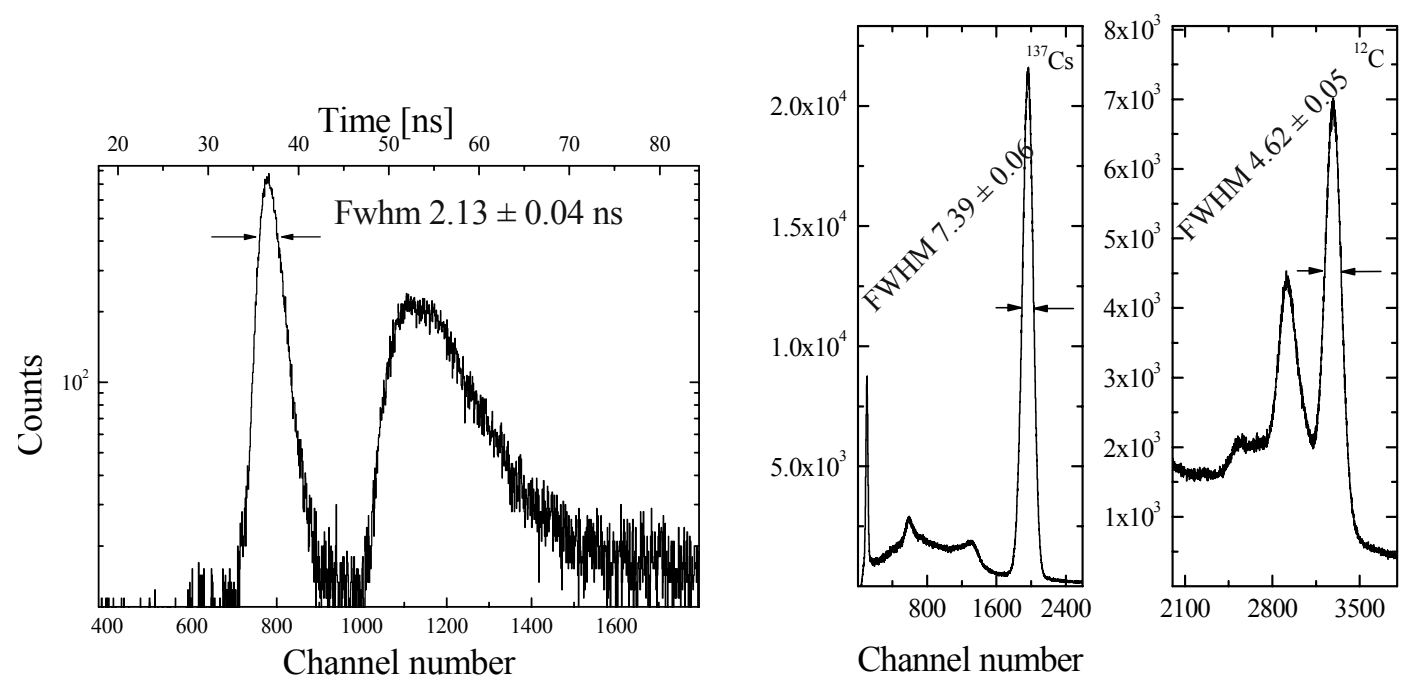

Channel number

Figure 11. Measured time (left) and pulse-height (right) spectra of the $5 " \times 5 " \times 10^{\prime \prime}$ sodium iodide detector. 


\section{ELECTRONICS}

The EURITRACK front-end electronics, adequately power supplied, manages the signals of all detectors, including the storage and the retrieval of all events. The power supply system is implemented with the CAEN SY2527 unit equipped with two A1733N boards (see Fig. 12) remotely controlled by a software interface integrated in the EURITRACK Information System (IS). The front-end electronics (FE) processes the signals of all detectors, performing the required coincidences between any pixel of the alpha particle detector and any gamma-ray and neutron scintillators. FE digitizes energy and time measurements of each event. The FE is composed of VME cards (see Fig. 12).

The block diagram of the acquisition system is drafted in Fig.13. After processing the individual anode signals by using Constant Fraction Discriminators (CAEN V812 16 channels boards), the programmable trigger unit (CAEN V1495) performs all the logic operations to build coincidences and determine the occurrence of a valid event. This card houses different Field-Programmable Gate Array (FPGA) units performing all required operations in short times. Once the valid event is ascertained, the anode signals from all gamma-ray detectors are digitized using Charge-to-Digital Converters (CAEN V792N QDC) to retrieve energy. The length of the integration gate is a compromise between energy resolution and count rate capability of the system. Elapsed time between any events can be recorded by a multi-hit Timeto-Digital Converter (CAEN V1190 TDC). Consequently it allows measurements of the delay between the produced gamma-ray and the associated alpha particle, but also identification of possible events with multiple alpha or gamma hits. These last might result in a loss of correlation in inspecting the suspect voxels.

The use of high density VME cards insures the compact size of the electronics. The FE is contained in a single VME crate, linked to the PC through the CAEN V2718/A2818 bridge. The V2718 card will be housed in the VME crate and the PCI card A2818 in the PC, data transmission being performed by an optical link. In the PC, the Data Acquisition System (DAQ) permits to store the events and build the spectra relevant for the inspection tasks. All FE and power supply settings and monitoring will be integrated in the EURITRACK IS through a user-friendly interface.
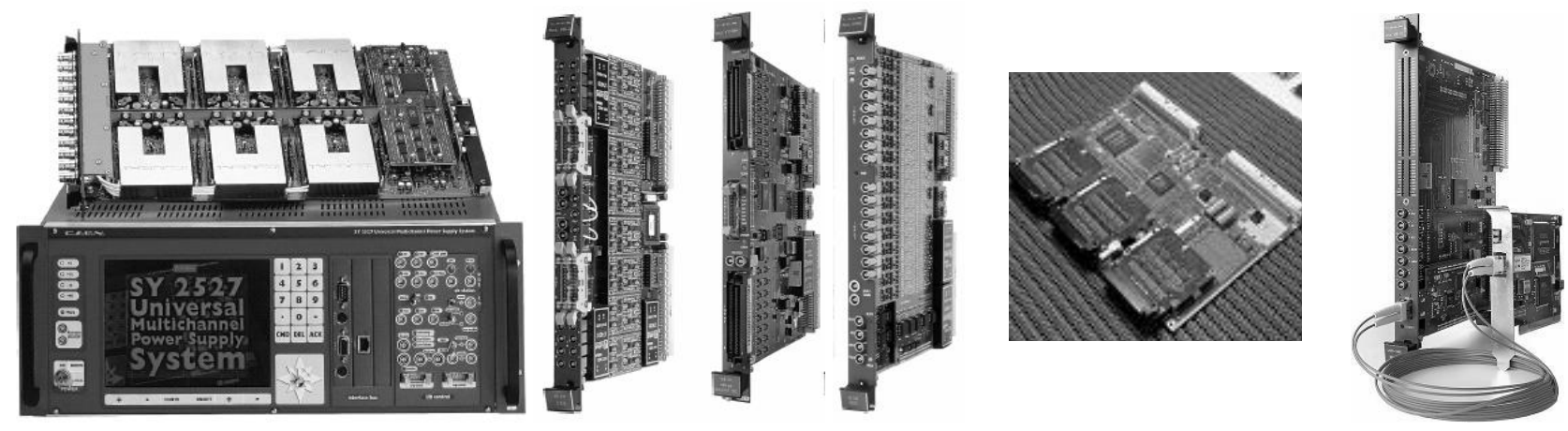

Figure 12. EURITRACK electronics. From left to right, power supply (CAEN SY2527 unit and A1733N board) and CAEN VME modules V812 CFD, V1190 TDC, V792N QDC, V1495 Trigger Unit (under development, shown without its front panel) and V2718/A2818 PCI bridge. 


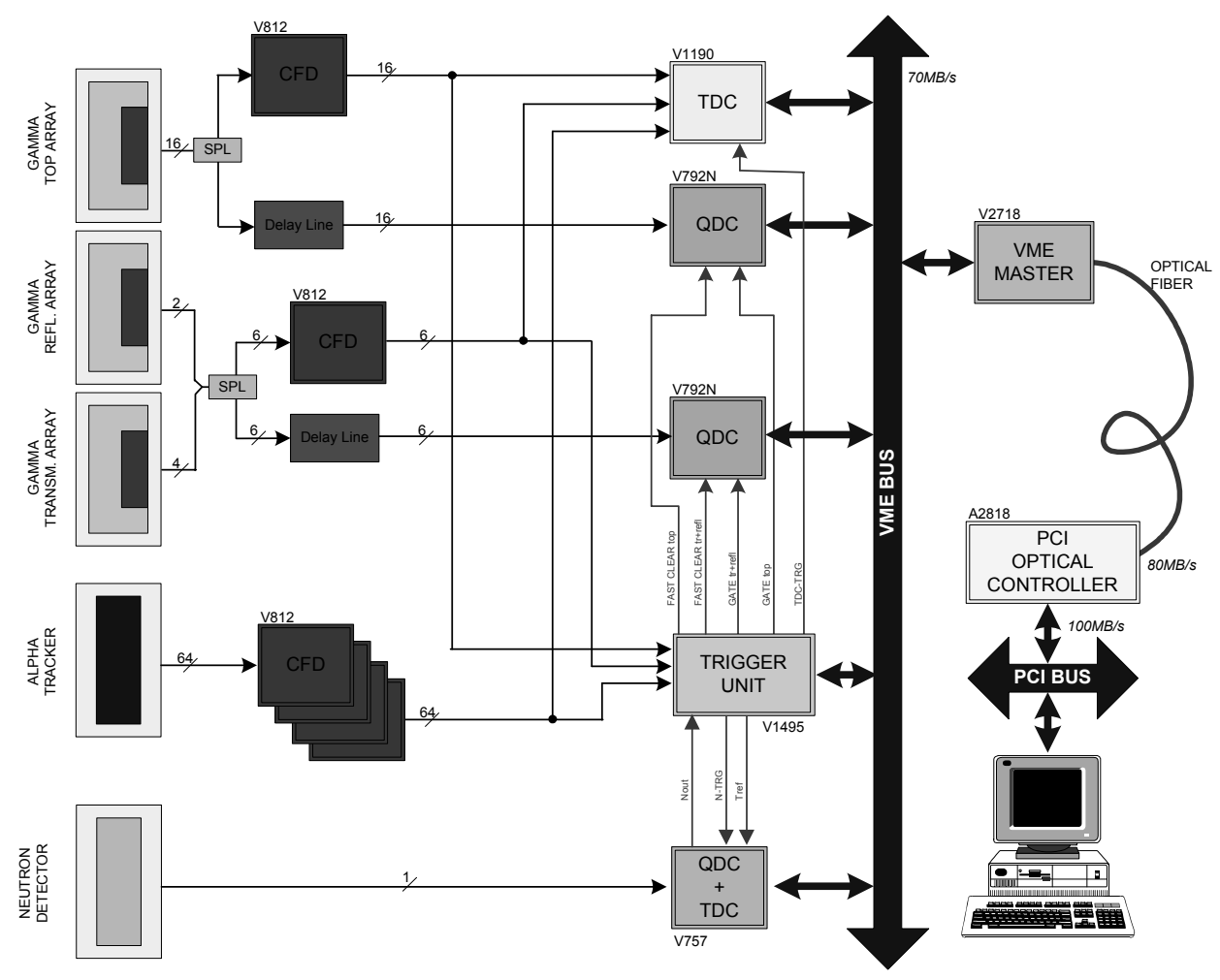

Figure 13. Block diagram of the EURITRACK front-end electronics.

\section{CONCLUSION}

The Tagged Neutron Inspection System (TNIS) has been designed by Monte Carlo calculations. Hardware components (associated particle neutron generator, detectors, electronics) have been developed and tested by the consortium members of the EURITRACK project, and will be integrated during the first part of year 2006 at the Ruder Boskovic Institute of Zagreb, Croatia. Data processing and decision-making software is being developed in parallel. The generator and detectors will be integrated on a mechanical portal at CEA Saclay, France, where the full system will be tested before the end of the year 2006. Finally, demonstration tests with will be performed during the year 2007 at the Seaport of Le Havre, in the area of the SYCOSCAN X-ray scanner operated by the French Customs.

\section{ACKNOWLEDGMENTS}

This work is supported by the European Union through the EURopean Illicit TRAfficking Countermeasures Kit project which is carried out in the framework of the specific research and technological development program "FP6-2003-IST-2 Proposal/Contract 511471.

\section{REFERENCES}

1. A. Buffler et al., "Material classification by fast neutron scattering" in Nucl. Instr. And Meth. B173, pp. 483-502, 2001.

2. T. Gozani, "The role of neutron based inspection techniques in the post 9/11/01 era", in Nucl. Instr. and Meth. B213, p. 460, 2004.

3. G. Vourvopoulos and P.C. Womble, "Pulsed fast/thermal neutron analysis: a technique for explosives detection", in Talanta 54, pp. 459-468, 2001.

4. C.M. Gordon, C.W. Peters, "A Fast-neutron Probe for Tomography and Bulk Analysis", in Appl. Radiat. Isot. Vol. 41, No. 10/11, pp. 1111-1116, 1990. 
5. J. F. Briesmeister, Editor, MCNP - A General Monte Carlo N-Particle Transport Code (Version 4C), LA-13079-M, Los Alamos National Laboratory, March 2000.

6. G. Nebbia, D. Fabris, M. Lunardon, S. Moretto, S. Pesente, G. Viesti, M. Cinausero, "Development of a Tagged Neutron Beams for the EURITRACK (EURopean Illicit TRAfficking Countermeasures Kit) Project", in IEEE Nuclear Science Symposium and Medical Imaging Conference, Puerto Rico, 23-29 October, 2005.

7. G. Perret, B. Perot, J-L. Artaud, A. Mariani, "EURITRACK Tagged Neutron Inspection System Design" in NPDC19 - $19^{\text {th }}$ Nuclear Physics Divisional Conference of the European Physical Society, Pavia (Italy), September 5-9, 2005.

8. J. Bialkowski, M. Moszynski and D. Wolski, "A pulse shape discriminator with high precision of neutron and $\gamma$ rays selection at high counting rate" in Nucl. Instr. and Meth. A280, p. 322, 1989.

9. Oe. Skeppsted, M. Moszynski, Z. Sujkowski, D. Wolski, M. Kapusta et al. "The EUROBALL neutron wall design and performance tests of neutron detectors" in Nucl. Instr. and Meth. A421, p.531, 1999.

10. M. Moszynski, G.J. Costa, G. Guillaume, B. Heusch, A. Huck and S. Mouatassim, "Study of n- $\gamma$ discrimination with NE213 and BC501A liquid scintillators of different size" in Nucl. Instr. and Meth. A350, p. 226, 1994.

11. M. Moszynski, G.J. Costa, G. Guillaume, B. Heusch, A. Huck and S. Mouatassim, "Comparative study of $130 \mathrm{~mm}$ diameter photomultipliers for neutron detectors" in Nucl. Instr. and Meth. A308, p. 97, 1991.

12. M. Gierlik, T. Batsch, M. Moszyński, D. Wolski, W. Klamra, B. Perot and G. Perret, "Comparative study of large $\mathrm{NaI}(\mathrm{Tl})$ and BGO scintillators for the EURopean Illicit TRAfficking Countermeasures Kit project", in IEEE Nuclear Science Symposium and Medical Imaging Conference, Puerto Rico, 23-29 October, 2005.

13. M. Moszynski, M. Kapusta, D. Wolski, M. Balcerzyk, S. O. Flyckt, P. Lavoute, C. Marmonier, and H. Mach, "New fast photomultipliers with a screening grid at the anode" in IEEE Trans. Nucl. Sci., vol. 51, No. 4, Aug. 2004.

14. T. Szczesniak, M. Gierlik, M. Kapusta, M. Moszynski, D. Wolski, P. Lavoute and E. Rossignol, "The $75 \mathrm{~mm}$ Diameter Photonis XP43D2 Photomultiplier with the Screening Grid at the Anode for Timing Experiments" in IEEE Nuclear Science Symposium \& Medical Imaging Conference, Puerto Rico, 23-29 October, 2005. 